\title{
Analysis of Wavelength Sensitivity of Coupling Coefficients and Inter-core Crosstalk in a 9 core Multi-core Fiber
}

Afzal Hossain ( $\square$ ayonarnab@yahoo.com )

Bangladesh University of Engineering and Technology

Satya Prasad Majumder

Bangladesh University of Engineering and Technology (BUET)

\section{Research Article}

Keywords: Capacity Crunch, Multi-Core Fiber, Coupling Coefficient, Mode Coupling, Power Coupling, Relative Crosstalk, Wavelength-dependent Crosstalk

Posted Date: November 8th, 2021

DOI: https://doi.org/10.21203/rs.3.rs-1036964/v1

License: (c) (i) This work is licensed under a Creative Commons Attribution 4.0 International License.

Read Full License 


\title{
Analysis of Wavelength Sensitivity of Coupling Coefficients and Inter-core Crosstalk in a 9 core Multi-core Fiber
}

\author{
Md Afzal Hossain and S P Majumder \\ Department of Electrical and Electronic Engineering (EEE) \\ Bangladesh University of Engineering and Technology (BUET) \\ Dhaka-1000, Bangladesh \\ ayonarnab@yahoo.com, spmajumder2002@yahoo.com
}

\begin{abstract}
Wavelength dependence of coupling coefficients and inter-core crosstalk in a 9-core homogeneous multi-core optical fiber (MCF) are investigated analytically. The analysis is further extended to evaluate the mean crosstalk power at the output of any core with light launched into other core of the MCF. Propagation length dependence of mean crosstalk power is investigated using both coupled mode theory (CMT) and coupled power theory (CPT). CPT based results show that mean crosstalk power linearly dependent on propagation distance, and it is higher for higher values of coupling coefficient. On the other hand, the mean crosstalk power is found to oscillate with the propagation distance in case of CMT. It is also observed that the mean crosstalk power $(\mathrm{dB})$ is more prominent at a lower wavelength for a given propagation distance. The behavour of relative crosstalk power is also investigated analytically where it is noticed that the relative crosstalk power increases almost linearly with core pitch and with wavelength. It is also seen that the relative crosstalk power (dB) is more in an MCF with lower number of cores when it is varied with respect to wavelength. This is due to the increase of core pitch under the same cladding diameter and cladding thickness limitations.
\end{abstract}

Keywords-Capacity Crunch, Multi-Core Fiber, Coupling Coefficient, Mode Coupling, Power Coupling, Relative Crosstalk, Wavelength-dependent Crosstalk

\section{INTRODUCTION}

During the recent decades, in the last decade in particular, the advent of streaming video transmissions and distributed and cloud computing have given an impetus to the requirement of transmission capacity growth [1]. This exponential growth of traffic demand has been fuelled by the emergence of new technologies and applications, like "Internet of Things (IoT)". Optical networks have been growing as well, with new technologies being implemented to keep pace with the increasing bandwidth demand with reduction of cost. [2]. Although the huge data traffic demand is met by wavelength-division multiplexed (WDM) systems implemented employing single-mode single-core fibers (SM-SCFs), recent studies find that the WDM systems are rapidly reaching their fundamental Shannon capacity limit, resulting in a state of capacity crunch of optical systems [3]. As on now the already implemented optical system solutions have used all the available domains (i.e, wavelength, time, polarization, amplitude and phase) but one that is space. This has encouraged the researchers in the field to explore the advanced optical fibers to replace the standard single mode fiber (SMF). Thus Space-Division Multiplexing (SDM) using Multicore fibers (MCFs) and Multi-mode fibers (MMFs) has opened up a new avenue in optical fiber communications to overcome the fundamental Shannon capacity limit [35]. In SDM technology, the fibers allow data transmission in parallel strands of MCF, a combination of multiple cores within a single cladding or multiple modes in a single core [6]. Leaving aside the ideal scenario, i.e., no change of refractive indices of core and cladding in optical fiber communication system, bending or stress whether intended or not, shall cause index perturbations in the fibers. This index perturbation will result in propagation of fields to evolve randomly causing coupling between signals of different modes or mode field of different cores [7, 8]. The coupling induced crosstalk will degrade the data transmission quality and limit the bandwidth capacity. Researches on SDM systems indicate that the crosstalk suppression has been a major concern in design and fabrication of MCFs for high-capacity long-distance transmission networks [9, 10]. Thus analysis of the coupling coefficient and resulting inter-core crosstalk is essential for designing any MCF based fiber optic communication system. Coupled-mode theory (CMT) and coupled-power theory (CPT) are introduced and studied extensively to evaluate and analyze the inter-core crosstalk in various MCFs $[11,12]$. By determining the coupling coefficients of the MCF, the mode coupling dynamics can be analyzed employing CMT directly or indirectly. In such efforts, mode coupling dynamics and communication strategies for 7 core MCF based communication systems was studied and presented in [13-15]. Study and analysis of 
wavelength-dependent inter-core crosstalk in MCFs appears important in designing and fabrication of future MCF based SDM-WDM transmission systems. To this effort wavelength dependence of inter-core crosstalk in homogeneous 7 core MCF is investigated and reported in [16]. Coupling coefficients of a 9 core homogeneous MCF are evaluated analytically and presented in [17]. In this paper, inspired by the potential applications of MCFs in SDM-WDM transmission systems, the wavelength sensitivity of coupling coefficients and resulting intercore crosstalk is analytically evaluated for various parameters of a 9 core MCF.

\section{PROPOSED FIBER STRUCTURE}

Fig.1. shows schematic of the cross section of a 9-core homogeneous MCF.

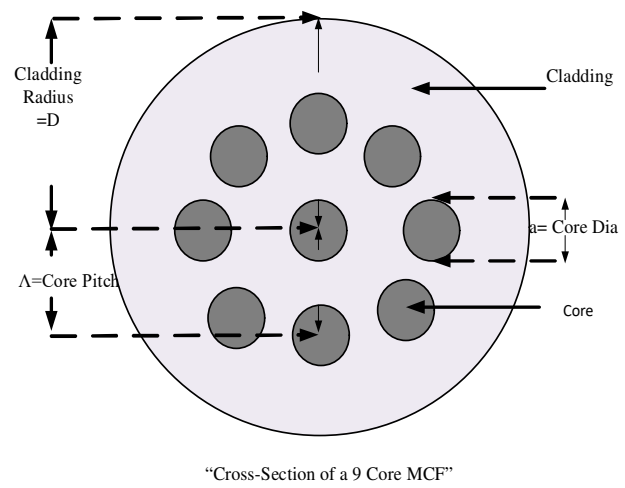

Fig. 1. Cross-section of a homogeneous 9-core Multicore Optical Fiber (MCF) structure

The cross section of a 9-core homogeneous MCF is considered to be consisted of a centre core (core-1) plus 8 other cores (cores 2 through 9). Each core has identical core parameters (refractive index, core pitch and radius). Table-1 presents the detail fiber parameters.

The numerical parameters of the cores of the MCF under investigation are shown in the Table 1.

Table -1 : Parameters of 9 Core MCF

\begin{tabular}{|l|l|l|}
\hline Symbol & Parameter & Value \\
\hline $\mathrm{n}_{\mathrm{c}}$ & Core Index & Varies with $\Delta$ \\
\hline $\mathrm{n}_{\mathrm{cl}}$ & Cladding Index & 1.444 \\
\hline $\mathrm{a}$ & Core radius & $2.50 \mu \mathrm{m}$ \\
\hline$\Lambda$ & Core-to-core distance & $\begin{array}{l}8.5 \mu \mathrm{m} \text { (if not varied } \\
\text { for Experiment) }\end{array}$ \\
\hline$\Lambda$ & Operating wavelength & $\begin{array}{l}1550 \mathrm{~nm} \mathrm{(if} \mathrm{not} \\
\text { varied for } \\
\text { Experiment ) }\end{array}$ \\
\hline$\Delta$ & Relative Index difference & $0.15 \%$ \\
\hline $\mathrm{R}_{\mathrm{b}}$ & Data rate & $5 \mathrm{Gbps}$ \\
\hline$D$ & Cladding Diameter & $125 \mu \mathrm{m}$ \\
\hline $\mathrm{R}_{\mathrm{bend}}$ & Bending Radius & $140 \mathrm{~mm}$ \\
\hline
\end{tabular}

\section{THE COUPLED-MODE EQUATIONS}

As shown in Fig.1, it is considered that there are $\mathrm{N}$ number of fiber cores (here, $\mathrm{N}=9$ ), and any core $\mathrm{m}$ is designated as $\mathrm{m}^{\text {th }}$ core with its radius $\mathrm{a}_{\mathrm{m}}$ and refractive index $n_{c m}$ respectively. The cladding refractive index is expressed as $n_{\mathrm{cl}}$. The cores are physically oriented maintaining distance in between adequate to ensure enough isolation enabling the use of perturbation methods with CMT for analysis of mode coupling mechanism [14].

The mode field of each core can be expressed as

$$
E_{m}(z)=A_{m}(\mathrm{z}) \mathrm{e}^{-\mathrm{j} \beta_{m} z}
$$

where, $A_{m}$ expresses the mode amplitude of the $\mathrm{m}^{\text {th }}$ core at a distance $z$ along along the fiber and $\beta_{m}$ indicates the propagation constant of the light in the single mode fiber core. Here, it is assumed that only $\mathrm{LP}_{01}$ (fundamental) mode propagates through each core of the MCF. A set of Coupled Mode Equations (CME) which can be written in matrix form as in [14] and shown in Eq. (2) can be used to describe the propagation of the mode fields in each core of the MCF:

$$
\frac{d A(\mathrm{z})}{d z}=-C A(\mathrm{z})
$$

where, $\mathrm{A}(\mathrm{z})=\left[A_{1}(\mathrm{z}) \mathrm{A}_{2}(\mathrm{z}) \mathrm{A}_{3}(\mathrm{z}) \ldots \ldots . . \mathrm{A}_{n}(\mathrm{z})\right]^{T}$ is a column vector; $\mathrm{T}$ denotes the transpose; $\mathrm{z}$ is the propagation distance in the direction of propagation and $\mathbf{C}$ is an $\mathrm{N} \times \mathrm{N}$ matrix. Here, $\mathrm{C}_{m n}$, the coupling coefficient between the cores $m$ and core $n$. Coupling coefficient is a measure of the spatial overlap of the mode fields of core $m$ and $n$ over the cross-sectional area of core $n$ [14].

Following reference [15], we can express the coupling coefficient, $\mathrm{C}_{\mathrm{mn}}$ in a step-index optical fiber as the mode field of core $m$ in terms of the local coordinate system of core $\mathrm{n}$ :

$$
C_{m n}=\left\{\begin{array}{c}
j C_{m n} \exp \left[j\left(\beta_{m}-\beta_{n}\right) z\right] \quad ; \quad m \neq n \\
0 \quad ; \quad m=n
\end{array}\right.
$$

where $\beta_{m}$ is the propagation constant of $L P_{01}$ mode for core $m . \mathrm{C}_{\mathrm{mn}}$ can be analytically determined as in [15]

$$
\begin{aligned}
& C_{m n}=\kappa\left(n_{1 m}^{2}-n_{2}^{2}\right) \frac{W_{m}^{2}}{n_{1 m} a_{m}^{2} V_{m}^{2} J_{1}^{2}\left(U_{m}\right)} \frac{J_{0}\left(U_{m}\right)}{\mathrm{K}_{0}\left(W_{m}\right)} \sqrt{\frac{\pi a_{m}}{2 W_{m} \Lambda}} \\
& \exp \left(-\frac{W_{m}}{a_{m}} \Lambda\right) \int_{0}^{a_{m}} J_{0}\left(\frac{U_{m}}{a_{m}} r\right) I_{0}\left(\frac{W_{m}}{a_{m}} r\right) r d r
\end{aligned}
$$

Where, $U_{m}^{2}=a_{m}^{2}\left(\kappa^{2} \mathrm{n}_{1 m}^{2}-\beta_{m}{ }^{2}\right)$ and $W_{m}^{2}=a_{m}^{2}\left(\beta_{m}{ }^{2}-\kappa^{2} n_{2}^{2}\right)$. $V_{m}=\kappa a_{m} n_{1 m}\left(2 \Delta_{1}\right)^{1 / 2}$ is the $\mathrm{V}$ number that determines the modes propagating in the $\mathrm{m}^{\text {th }}$ core; $J_{1}\left(U_{m}\right)$ is the Bessel function of the $1^{\text {st }}$ kind with $1^{\text {st }}$ order and $\mathrm{K}_{0}(\mathrm{Wm})$ is the modified Bessel function of the $2^{\text {nd }}$ kind with zero order; $\kappa$ is the wave number in free space expressed as $\kappa=\frac{2 \pi}{\lambda}, \lambda$ is the wavelength of the light travelling in free space; $\Lambda$ is the pitch (from centre core to the outer 
cores). In the following sections, we analytically examine the characteristics of coupling coefficients and crosstalk induced due to the variation of physical parameters of the cores in the MCF. Our analysis is further extended to determine the wavelength-sensitivity as well as propagation length dependence of the coupling induced crosstalk power in the 9 core MCF.

\section{EVALUATION OF COUPLING COEFFICIENTS}

Researcers and engineers find it challenging to reduce the inter-core and intra-core coupling induced crosstalk for design and fabrication of coupled core MCF based optical fiber communication system. Most of the research works with MCF focus on technique to reduce this crosstalk [15].

In this paper, to investigate the mode coupling dynamics in a 9-core homogeneous MCF, we use the CMT and CPT as presented in [13-15]. The solution to the coupled-mode Eq. (2) for evaluating the numerical values of the coupling coefficients is obtained from the mathematical model given in Eq. (4). We have solved the Eq. (4) using MATLAB for the parameters given in Table 1. Now, according to the grouping of the cores based on their core-to-core separation and geometric orientation, the coupling coefficient, $\mathrm{C}_{\mathrm{mn}}$ matrix can be written as in [17] :

$$
C=j \times\left[\begin{array}{ccccccccc}
0 & C_{12} & C_{12} & C_{12} & C_{12} & C_{12} & C_{12} & C_{12} & C_{12} \\
C_{12} & 0 & C_{23} & C_{24} & C_{25} & C_{26} & C_{25} & C_{24} & C_{23} \\
C_{12} & C_{23} & 0 & C_{23} & C_{24} & C_{25} & C_{26} & C_{25} & C_{24} \\
C_{12} & C_{24} & C_{23} & 0 & C_{23} & C_{24} & C_{25} & C_{26} & C_{25} \\
C_{12} & C_{25} & C_{24} & C_{23} & 0 & C_{23} & C_{24} & C_{25} & C_{26} \\
C_{12} & C_{26} & C_{25} & C_{24} & C_{23} & 0 & C_{23} & C_{24} & C_{25} \\
C_{12} & C_{25} & C_{26} & C_{25} & C_{24} & C_{23} & 0 & C_{23} & C_{24} \\
C_{12} & C_{24} & C_{25} & C_{26} & C_{25} & C_{24} & C_{23} & 0 & C_{23} \\
C_{12} & C_{23} & C_{24} & C_{25} & C_{26} & C_{25} & C_{24} & C_{23} & 0
\end{array}\right]
$$

Here, with a close look into the fiber cross-section as shown in Fig. 1, it is clear that the closest cores are: Core2-core3; core3-core4; core4-core5, ...., core8core2. Solving the Eq. (4) using MATLAB we obtain the values of the coupling coefficients between the cores. The coupling coefficient of this group of cores is expressed as $\mathrm{C}_{23}$. For example, the value of $\mathrm{C}_{23}$, the coupling coefficient between the closest adjacent cores (2 and 3 ) is obtained as $0.0833(1 / \mathrm{m})$ with the core parameters as shown in Table 1. The next nearest distant cores are the cores from the centre core (core 1) to each of the peripheral cores (core 2 through core 8), and the distance is $\Lambda$. The coupling coefficient of this group of cores is expressed as $\mathrm{C}_{12}$. Similarly, the next distant cores are: core2-core4; core3-core5; core4core6, core5-core7, core6-core8. The coupling coefficient of this group of cores is expressed as $\mathrm{C}_{24}$. Similarly the coupling coefficient of the next larger distant group of cores is expressed as $\mathrm{C}_{25}$, and the coupling coefficient of the farthest distant cores (core2core 6 and so on) is expressed as $C_{26}$. The value of $C_{26}$, coupling coefficient between the farthest cores (2 and 6) is obtained as $0.002(1 / \mathrm{m})$.

\section{ANALysis of INTER-CORE Crosstalk}

When light is launched into the center core (core-1), that means $A_{1}(0)=1$ and $A_{m}(0)=0$ for $m \neq 1$, solutions for the normalized mode power in various cores of the 9 core MCF are expressed as in [14]:

$$
\begin{gathered}
\left|\mathrm{A}_{1}(\mathrm{z})\right|^{2}=\frac{1}{9}+\frac{8}{9} \cos ^{2}\left(\sqrt{9} \mathrm{C}_{23} \mathrm{z}\right) \quad \text { (6) and } \\
\left|\mathrm{A}_{\mathrm{m}}(\mathrm{z})\right|^{2}=\frac{1}{9} \sin ^{2}\left(\sqrt{9} \mathrm{C}_{23} \mathrm{z}\right) \quad \mathrm{m} \neq 1 \quad \text { (7) }
\end{gathered}
$$

where, $\mathrm{C}_{23}$ is the coupling coefficient between core-2 and core-3 (the closest adjacent cores).

The minima of $\left|A_{1}(z)\right|^{2}$ (normalized mode power) are located at the maxima of $\left|A_{m}(z)\right|^{2}$. As per Eq. (13) of [14], after doing some algebraic manipulations, it is obtained that the normalized mode power drops to its first minimum at a distance $L C$, given by

$$
L C=\frac{\pi}{2 \sqrt{N} C_{m n}},
$$

where, $N$ is the number of cores in the MCF $(N=9$, in this case) and $L C$ is the coupling length. By definition, the propagation length at which the normalized mode power in the $m^{\text {th }}$ excited core drops from one to its first minimum is termed as generalized coupling length. It serves as a measure for evaluating the coupling strength of composite waveguides such as MCFs [14].

Because of inter-core coupling, mode power of $\mathrm{m}^{\text {th }}$ core will also enter the adjacent cores. Here we have evaluated the coupling coefficients using Eq. (4), and taken the largest value (coupling between the closest adjacent cores) of the same (worst case scenario) in all our calculations. Crosstalk (Xtalk) power is evaluated using the two equations- Eq. (19) and Eq. (30) based on CMT and CPT respectively. After performing some algebraic simplifications crosstalk in core 2 due to core-3 can be expressed as [17]:

$$
\begin{aligned}
& (\mathrm{Xtalk})_{\mathrm{CM} \mathrm{T}}=\frac{\frac{1}{9} \sin ^{2}\left(\sqrt{9} \mathrm{C}_{23} \mathrm{z}\right)}{1-\frac{8}{9} \sin ^{2}\left(\sqrt{9} \mathrm{C}_{23} \mathrm{z}\right)} \\
& (\mathrm{X} \text { talk })_{\mathrm{CPT}}=\frac{1-\exp \left(-9 \mathrm{C}_{23} \mathrm{z}\right)}{1+8 \exp \left(-9 \mathrm{C}_{23} \mathrm{z}\right)}
\end{aligned}
$$

The statistical mean of Xtalk between two adjacent cores of a homogeneous MCF can be expressed as [16]

$$
\text { Xtalk }_{\text {mean }}=\frac{2 C_{23}^{2} R_{\text {bend }}}{\beta_{m} \Lambda} z
$$


The difference of Xtalk in $\mathrm{dB}$ between any wavelength $\lambda$ and the bottom end of $C$ and $L$ band wavelength $\lambda_{0}$ (i.e. $1530 \mathrm{~nm}$ ) is termed as relative Xtalk, and can be expressed as [16]

$$
\Delta \text { Xtalk }_{d B}=10 \log _{10} \frac{X \text { talk }_{\text {mean }}^{\lambda}}{X_{\text {talk }}^{\lambda_{\text {mean }}}}=10 \log _{10}\left[\left(\frac{C_{m n}^{\lambda}}{C_{m n}^{\lambda_{0}}}\right)^{2} \frac{\beta_{\lambda_{0}}}{\beta_{\lambda}}\right]
$$

where Xtalk mean $^{\lambda}, X_{\text {talk }}^{\lambda_{0}}, C_{m n}^{\lambda}, C_{m n}^{\lambda_{0}}, \beta_{\lambda}$ and $\beta_{\lambda_{0}}$ are the mean Xtalk, mode coupling coefficients, and propagation constants at a wavelength of $\lambda$ and $\lambda_{0}$, respectively. The above Eq. (12), upon some algebraic manipulation can be approximated and expressed as [16]

$\Delta$ talk $_{d B} \approx 10 \log _{10}\left[Q+\exp \left(2.2856 \frac{C}{\lambda_{0}} \frac{\lambda-\lambda_{0}}{\lambda} \frac{\Lambda}{r_{c}}\right)\right]$

where $C=2 \pi a n_{c}(2 \Delta)^{\frac{1}{2}}, Q$ is a coefficient that depends on $C$ and the operating wavelength $\lambda$, and can be approximated to be $(1-0.001256 \Delta \lambda)^{4}$ with an acceptable accuracy of $\pm 0.3 \%, \Delta_{\lambda}=\lambda-\lambda_{0}$. Eq. (13) can be rewritten as

$$
\Delta \text { talk }_{d B} \approx 10 \log _{10} Q+19.85 \pi n_{c} \sqrt{2 \Delta} \frac{\Delta \lambda}{\lambda \lambda_{0}} \Lambda
$$

The slope of the relative Xtalk (in $\mathrm{dB} / \mathrm{nm}$ ), a measure of the rate of change of relative Xtalk per unit wavelength $(/ \mathrm{nm})$ in normal step-index MCFs can be determined as

$$
\begin{aligned}
& \frac{d\left(\Delta \text { talk }_{d B}\right)}{d \lambda}=M=\frac{\Delta \text { talk }_{d B}}{\Delta \lambda} \\
& =\frac{10 \log _{10} Q}{\Delta \lambda}+19.85 \pi n_{c} \sqrt{2 \Delta} \frac{\Lambda}{\lambda \lambda_{0}}
\end{aligned}
$$

\section{Results And Discussions}

We have used COMSOL Multiphysics with MATLB (ver. 5.5a) simulation software to determine the effective refractive index of the cores of the 9 core homogeneous MCF for the fundamental $\mathrm{Lp}_{01}$ mode, which, in turn is used to determine the propagation constant in that particular core of the MCF. Fig.2. depicts the mode (Lp $\left.p_{01}\right)$ field distribution in the cores of the MCF.
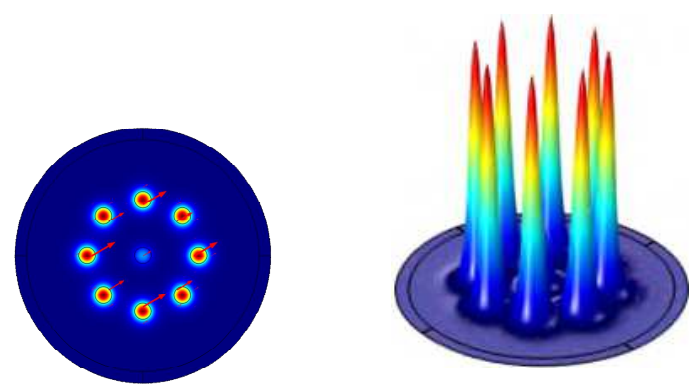

Fig.2. Plots (surface and arrow plots) of mode fields in the cores of a homogeneous 9 core MCF with given wavelength $(1.55 \mu \mathrm{m})$, relative refractive index $(0.0015)$, core radius $=2.5 \mu \mathrm{m}$ and core pitch $=8.5 \mu \mathrm{m}$
In Fig.3., the relationship between the normalized mode power and transmission distance, $z$ in a homogeneous 9-core MCF as obtained from Eq. (6) and Eq. (7) is depicted when light is injected into the centre core with $\Delta=0.0015$; core radius $\mathrm{a}_{\mathrm{m}}=2.5 \mu \mathrm{m}$; core pitch, $\Lambda=8.5$ $\mu \mathrm{m}$ and bending radius $R_{\text {bend }}=140 \mathrm{~mm}$. It is observed that the normalized mode power is periodic with propagation distance $z$, and the maxima of the mode power of other core (here, core 2) lies in the minima of the excited core (here core 1).

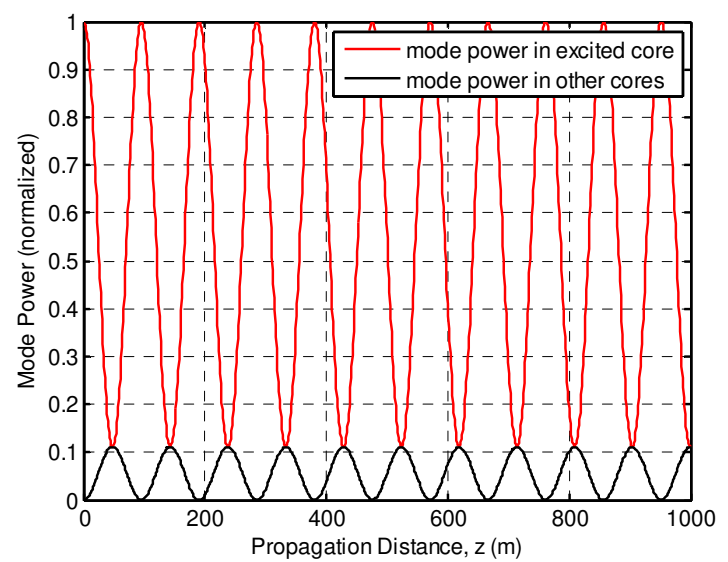

Fig.3. Variation of mode power (normalized) in the excited core and other cores in homogeneous 9 core MCF at given wavelength $(1.55 \mu \mathrm{m})$, relative refractive index $(0.0015)$, core radius $(2.5 \mu \mathrm{m})$ and core pitch $(8.5 \mu \mathrm{m})$

The variation of coupling coefficients with respect to relative refractive index difference, $\Delta$ at various core pitches is depicted in Fig. 4. It is observed that the coupling coefficient decreases with the increase of relative refractive index contrast. The figure also shows that more coupling occurs (higher coefficient) at the lower values of core pitch within the same cladding radius.

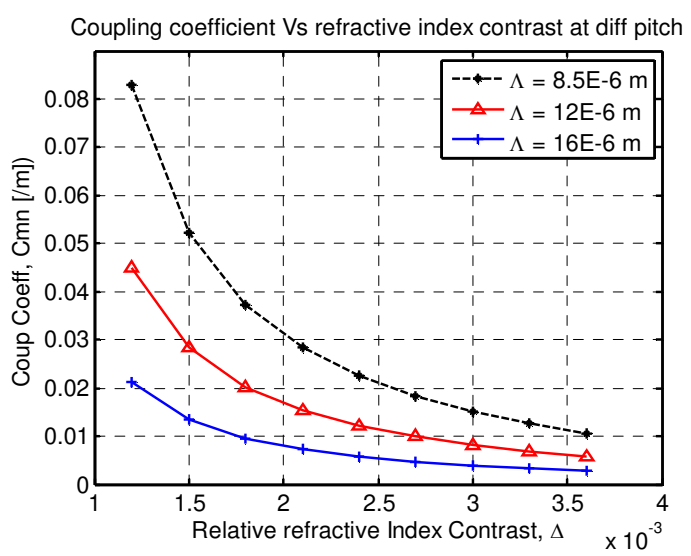

Fig. 4. Variation of coupling coefficient with respect to refractive index contrast, $\Delta$ in 9 core homogeneous MCF at a given wavelength $(1.55 \mu \mathrm{m})$, core radius $(2.5 \mu \mathrm{m})$ and at various core pitch

Fig.5. depicts the relationship of coupling coefficients of different cores with respect to the operating wavelength. It is noticed that with the increase of wavelength, coupling coefficient increases, i.e, there lies almost a linear relationship between the coupling coefficient and 
the wavelength. It is also seen that increase in coupling coefficient is higher for the closest adjacent cores (here $\mathrm{C}_{23}$ ) and lower for the distant cores.

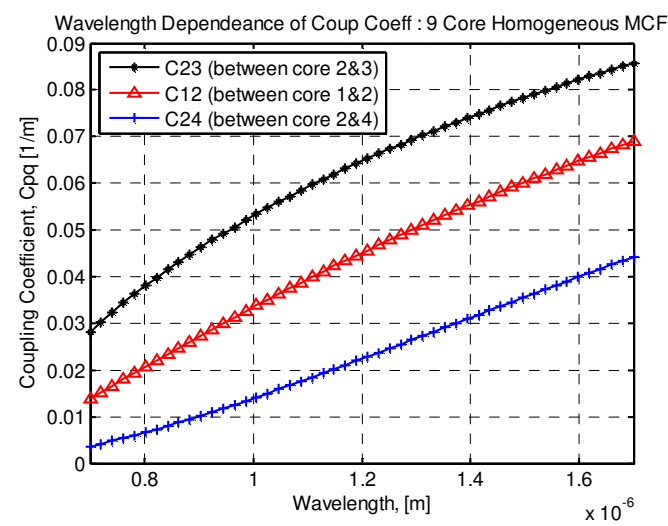

Fig. 5. Variation of coupling coefficient with respect to the operating wavelength in a 9 core homogeneous MCF with a given relative refractive index (0.0015), core radius $(2.5$ $\mu \mathrm{m})$ and core pitch $(8.5 \mu \mathrm{m})$

Fig.6. presents the relationship of coupling length (for the fundamental mode in the centre core coupled to the outer cores) with respect to the operating wavelength. It is noticed that with the increase of the wavelength, the coupling length decreases for all the cores. It is also seen that the rate of decrease in coupling length is higher for the farthest cores $\left(\mathrm{C}_{25}\right.$, in this case) and lower for the closest core $\left(\mathrm{C}_{23}\right.$, in this case).

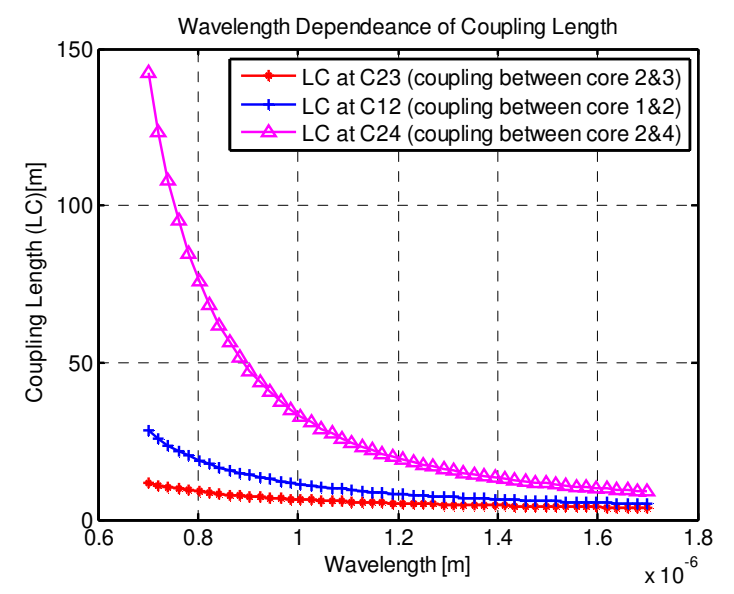

Fig. 6. Variation of coupling length with respect to the operating wavelength in a 9 core homogeneous MCF with a given relative refractive index $(0.0015)$, core radius $(2.5 \mu \mathrm{m})$ and core pitch $(8.5 \mu \mathrm{m})$

Fig.7. shows the length dependence of crosstalk power in a 9 core homogeneous MCF. The calculation is performed with a given wavelength $(1.55 \mu \mathrm{m})$, relative refractive index contrast $(0.0015)$, core radius $(2.5 \mu \mathrm{m})$ and core pitch $(8.5 \mu \mathrm{m})$. Assuming the direct detection at receiver end the calculation is done based on Coupled Mode Theory (CMT). MATLAB software is used to evaluate the crosstalk using the Eq. (19) of [12] where the highest coupling coefficient $\left(\mathrm{C}_{23}=0.0833\right)$ is used. It is noticed that the crosstalk power oscillates with varying amplitude and short period of length.

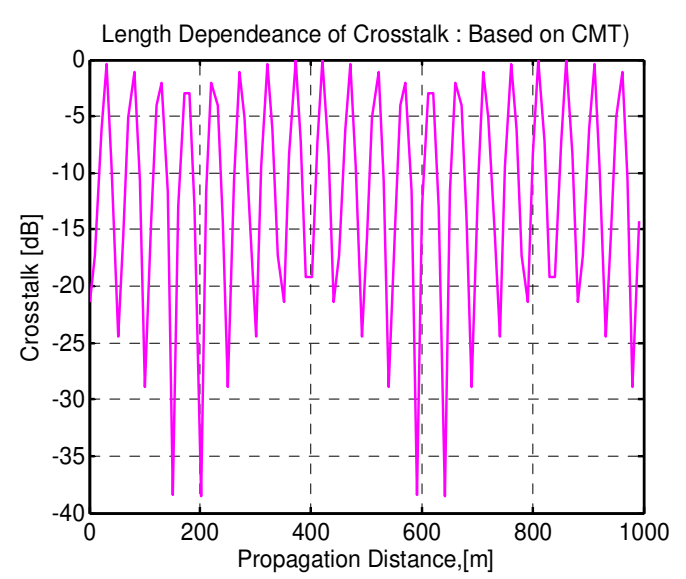

Fig. 7. Crosstalk power variation with respect to propagation distance in a homogeneous 9 core MCF at given wavelength $(1.55 \mu \mathrm{m})$, relative refractive index contrast $(0.0015)$, core radius $(2.5 \mu \mathrm{m})$ and core pitch $(8.5 \mu \mathrm{m})$ based on Coupled Mode Theory (CMT)

Fig.8. shows the propagation length dependence of crosstalk power in a 9 core homogeneous MCF. Here, the calculation is performed with the fiber parameters as given in Table 1, and based on Coupled Power Theory (CPT) considering random fluctuation on each core in the longitudinal direction (missing coherence) due to bending and stress for obvious reasons. MATLAB software is used to evaluate the crosstalk using the Eq. (30) of [12] where the power coupling between the adjacent cores (closest adjacent cores) as well as the same between other distant cores are evaluated using their corresponding coupling coefficients. It is noticed that the crosstalk power increases with the increase of propagation distance $(z)$, and it is higher with the higher coupling coefficient.

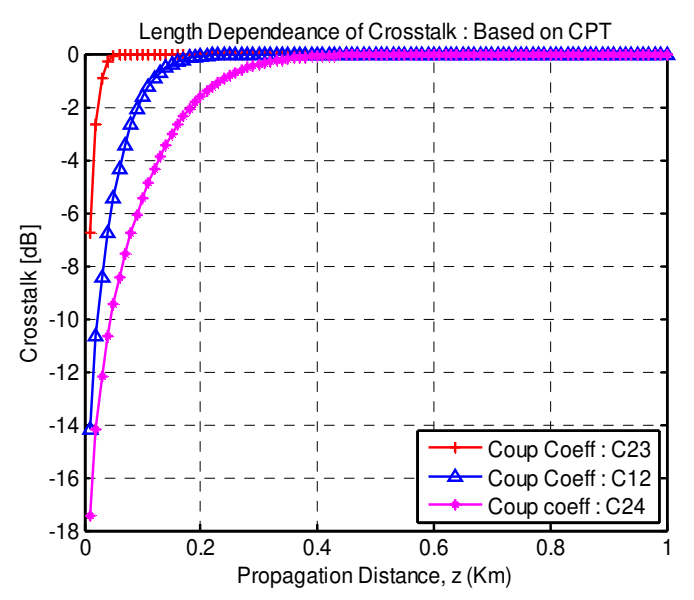

Fig.8. Variation of crosstalk power in the cores of a homogeneous 9 core MCF with respect to propagation distance at a given wavelength $(1.55 \mu \mathrm{m})$, relative refractive index $(0.0015)$, core radius $(2.5 \mu \mathrm{m})$ and core pitch $(8.5 \mu \mathrm{m})$ based on Coupled Power Theory (CPT)

Fig.9. depicts the wavelength-dependence of crosstalk power in a 9 core homogeneous MCF. Here, the calculation is performed with the fiber parameters as given in Table 1, and based on Eq. (10) wherein the power coupling between the adjacent cores is only investigated considering the worst case scenario. It is noticed that the mean crosstalk power increases with the 
increase of operating wavelength at a particular propagation distance $(\mathrm{z})$, and it is higher with the higher propagation distance.

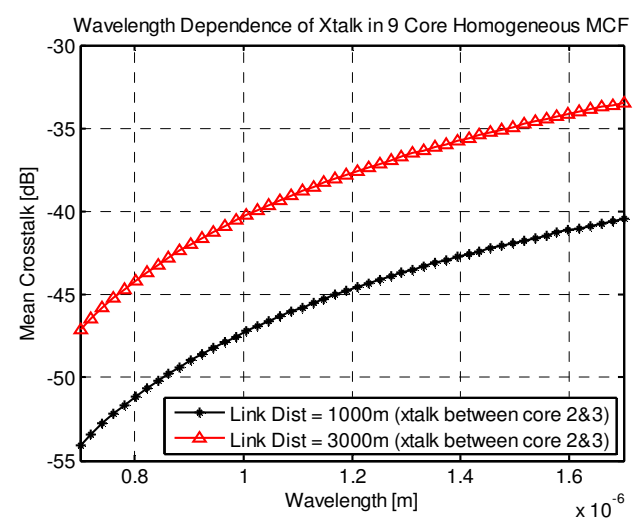

Fig. 9. Wavelength dependence of crosstalk power in the cores of a homogeneous 9 core MCF with respect to the operating wavelength $(\lambda)$ at a given propagation distance $(z)$, relative refractive index $(0.0015)$, core radius $(2.5 \mu \mathrm{m})$ and core pitch $(8.5 \mu \mathrm{m})$

Fig. 10. shows the comparison of relative crosstalk (in $\mathrm{dB}$ ) in 7 and 9 core homogeneous MCFs with corresponding core pitches of $8.5 \mu \mathrm{m}$ and $10.5 \mu \mathrm{m}$ within a wavelength span of $C$ and $L$ band $(1530 \mathrm{~nm}$ to 1620 $\mathrm{nm})$ respectively. The result shows that the relative crosstalk in the MCF with more number of cores is less as compared to that in the MCF with less number of cores. This is due to the reduced core pitch of MCF with more number of cores under the same cladding diameter and cladding thickness constraints. Our analytical result is in agreement with the results presented in [16, Fig.4.].

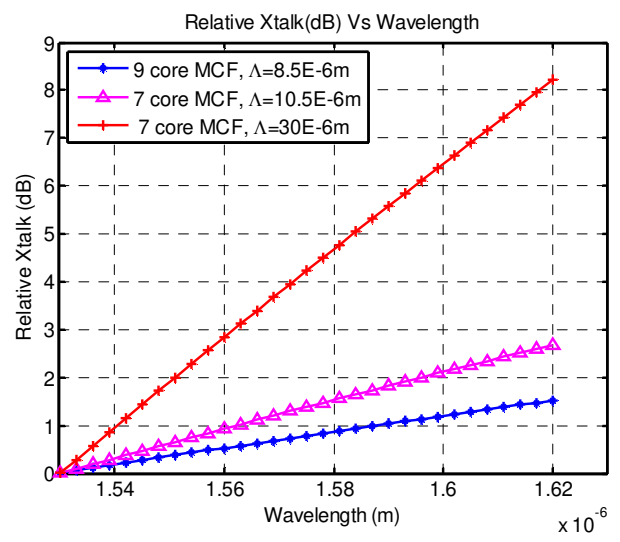

Fig. 10. Comparison of relative crosstalk in 7 and 9 core homogeneous MCFs with corresponding core pitches of $8.5 \mu \mathrm{m}$ and $10.5 \mu \mathrm{m}$ within a wavelength span of $C$ and $L$ band (1530 nm to $1620 \mathrm{~nm})$ and relative refractive index 0.0015 .

Fig. 11. depicts the comparison of relative crosstalk $(\mathrm{dB})$ in 9 core homogeneous MCF with respect to the variation of core pitch at different relative refractive indices and at an operating wavelength of $1620 \mathrm{~nm}$. It is seen from the figure that the relative crosstalk shows almost a linear relationship with the core pitch at different relative refractive indices. It is also noticed that the relative crosstalk is higher for the higher value of relative refractive index. Our analytical finding is in agreement with the results presented in [16, Fig.5.].

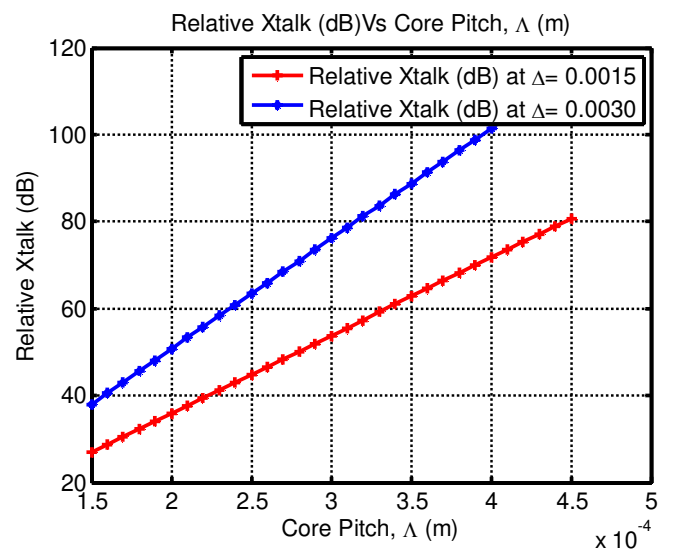

Fig. 11. Comparison of relative crosstalk in 9 core homogeneous MCF with respect to the variation of core pitch at different relative refractive indices at an operating wavelength of $1620 \mathrm{~nm}$ and over a wavelength span of $C$ and $L$ band (1530 nm to $1620 \mathrm{~nm}$ ).

\section{CONCLUSION}

In this paper, the coupling coefficients among various cores in a 9-core homogeneous MCF are evaluated analytically. The relationship of the coupling coefficients with respect to core pitch, relative refractive index difference and operating wavelength are determined and discussed. A trend of decrease of the coupling coefficient with the increase of core pitch, $\Lambda$ and relative refractive index contrast, $\Delta$ is observed. On the contrary, the coupling coefficient increases exponentially with the increase of operating wavelength, $\lambda$. Inter-core coupling induced crosstalk is evaluated analytically using both CMT and CPT. The length-dependence as well as wavelength-dependence characteristics of the crosstalk power are investigated and presented in this paper. Both statistical mean crosstalk and relative crosstalk are investigated here. Analysis shows that the mean crosstalk increases with the increase of operating wavelength. The CPT is found to be more realistic to evaluate and analyze the crosstalk power in MCF because it takes into account the random fluctuation on each core. The outcome of this research may find its application in design and fabrication of MCFs for implementing SDM-WDM optical communication systems with MCF to meet the ever-increasing demand of transmission channel capacity.

\section{ACKNOWLEDGEMENTS}

The authors acknowledge the support provided by the department of EEE, BUET to conduct this research as a part of the dissertation.

\section{References:}

[1] R. W. Tkach, "Network Traffic and system capacity: scaling for the future," In: $36^{\text {th }}$ European Conference and Exhibition on Optical Communication (ECOC), 2010, pp. 1-22

[2] D. Richardson, J. Fini and LE. Nelson, "Spacedivision multiplexing in optical fibres," Nature Photonics. 2013, vol. 7: pp. 354-362 
[3] Van Uden RGH et al. "Ultra-high-density spatial division multiplexing with a few-mode multi-core fibres," Nature Photonics. 2014, vol. 8: pp. 865-870

[4] S. Inao, T. Sato, S. Sentsui, T. Kuroha and Y. Nishimura, "Multi-core optical fiber," In: The Optical Fiber Communication Conference and Exhibition (OFC). Washington: Optical Society of America; 1979. WB1

[5] S. Berdagué and P. Facq, "Mode-division multiplexing in optical fibers," Applied Optics. 1982, vol. 21(11), pp. 1950-1955

[6] B. J. Puttnam, S. L. Ruben, G. Rademacher, A. Alfredsson, K. Werner, J. Sakaguchi, Y. Awaji, E. Agreel and N. Wada, "Characteristics of homogeneous multicore fibers for SDM transmission," APL Photonics 4, 022804 (2019); pp. 1-10. (newly added and referred to in the text)

[7] K. Saitoh and M. Matsuo, "Multicore Fiber Technology," Journal of Lightwave Technology. 2016, vol. 34(1): pp. 55-66

[8] S Matsuo, K. Takenaga, Y. Arakawa, Y. Sasaki, S. Tanigawa, K. Saitoh, and M. Koshiba, "Crosstalk behavior of cores in multi-core fiber under bend condition," IEICE Electron. Express, 2011, vol. 8(6) : pp. 385-390

[9] T Mizuno, H. Takara, A Sano and Y. Miyamoto, "Dense space-division multiplexed transmission systems using multi-core and multi-mode fiber," Journal of Lightwave Technology. 2016, vol. 34(2): pp. 582-591

[10] K. Imamura, Y. Tsuchida, K. Mukasa, R. Sugizaki, K. Saitoh, and M. Koshiba, "Investigation on multi-core fibers with large Aeff and low micro bending loss," Opt. Express , 2011, vol. 19(11) : pp. 10595-10603

[11] A. W. Snyder, "Coupled mode Theory for Optical fibers," Optical Society of America. 1972, vol. 62(11): pp. 1267-1277

[12] K. Takenaga, Y. Arakawa, S. Tanigawa, N. Guan, S. Matsuo, K. Saitoh, and M. Koshiba, "An investigation on Crosstalk in Multicore Fibers by Introducing Random Fluctuation along Longitudinal Direction," IEICE Trans. Commun. 2011, vol. E94-B : pp. 409-416

[13] W. Ren and Z. Tan, "A study on the coupling coefficients for multicore fibers," Optik - International Journal for Light and Electron Optics, 2016, vol. 127 (6) : pp. $3248-3252$

[14] F. Y. M. Chan, A. P. T. Lau, and H.-Y. Tam, "Mode coupling dynamics and communication strategies for multi-core fiber systems," Opt. Express, 2012, vol. 20 : pp. 4548-4563

[15] Ye Feihong, Tu. Jiajing, et al. "Simple analytical expression for crosstalk estimation in homogeneous trench-assisted multi-core fibers," Optics Express, 2014, vol. 22 (19) : pp 23007- 23018

[16] Y. Feihong, T. Jiajing et al., "Wavelength dependence of Inter-Core Crosstalk in Homogeneous Multi-Core Fibers," IEEE PHOTONICS TECHNOLOGY LETTERS, 2016, vol. 28 (1), pp. 27-30

[17] M. A. Hossain and S. P. Majumder, "Analytical Evaluation of Coupling Coefficients of a Nine-core Homogeneous Optical Fiber," International Journal for Light and Electron Optics (Optik, Elsevier), 2020 (200):

ID: IJLEO_163204, pp: awaiting printing) 